\title{
Über die Regeneration des extrahierten Sehpurpurs.
}

\author{
Von \\ Yuji Hosoya und Tomio Sasaki. \\ (細谷雄二) (佐令木富雄) \\ (Aus dem Physiologischen Institut von Prof. T. Fujita, \\ Kaiserliche Tohoku-Universität zu Sendai.)
}

Es wurde von $K \ddot{u} h n \mathrm{e}^{1)}$, Garten ${ }^{2)}$ und Andogrk $y^{3)}$ festgestellt, dass für die Regeneration des Sehpurpurs in der Retina die Verbindung oder Berührung des Sehepithels mit dem Pigmentepithel eine notwendige Bedingung ist, indem sich der Sehpurpur vom ausgebleichten $\mathrm{Zu-}$ stand, d. h. vom Sehgelb resp. Sehweiss durch die Zufuhr gewisser Substanzen (Rhodophylin nach Ki ühne) aus dem Pigmentepithel wiederherstellt. Küh ne fand ähnliche Regeneration auch beim extrahierten Sehpurpur, und Garten, der die Regeneration in der isolierten Netzhaut beobachtete, hielt dieselbe auch im extrahierten Zustand für möglich. Hos o y a ${ }^{4)}$ untersuchte aber die spektrale Absorption und die Farbenveränderung der Sehpurpurextrakte, die sowohl durch glykocholsaures Natron oder Digitonin wie auch Saponin, Panaxtoxin, oleinsaures Natron hergestellt wurden, vor und nach der Lichteinwirkung und fand, dass, wenn die Bleichung bis zum Sehgelb vorgeschritten war, keine Regeneration stattfand, auch wenn es im Dunkeln gehalten wurde, sondern die Bleichung noch weiter fortschritt. Diese letztere von ihm als die „Nachblejchung" bezeichnete Erscheinung wurde von $\mathrm{Hecht}{ }^{5)} \mathrm{Wald}^{(6)} \mathrm{u}$. a. bestätigt. Also ist der $\mathrm{K} u \mathrm{u} h n$ esche Befund der Sehpurpurregeneration im Extrakt lange unbestätigt geblieben, bis es erst ganz neulich $\mathrm{Hecht}$ und seinen Mitarbeitern ${ }^{5}$ zum ersten Male gelang, die Regeneration in der Extraktflüssigkeit zu konstatieren.

Nun erhebt sich die wichtige Frage, ob die Regeneration des Sehpurpurs im Extrakt, falls sie überhaupt möglich, nur beim Vorhandensein gewisser aus dem Pigmentepithel stammender Stoffe stattfindet oder auch ohne sie geschehen kann.

Die Dunkelnetzhaut des Frosches, die als beliebtes Material für die Sehpurpurdarstellung gebraucht wird, ist von der Chorioidea und 
Pigmentepithelschicht leicht abpräparierbar. Und da dabei die in den Fortsätzen der Pigmentzellen enthaltenen Substanzen, die sog. Fuszin körner, zum grössten Teil an der Chorioidea anhaften bleiben, sieht die Netzhaut makroskopisch pigmentfrei aus. Mikroskopisch dagegen können wir an ihr in der Regel nicht nur bazillenförmige Fuszinkörner, sondern auch viele Pigmentzellen mit eigenem hexagonal-mosaischem * Bild nachweisen. Dies gilt, wenn die Netzhaut unter dem hellen Tageslicht beobachtet wird ; geschweige denn bei der Netzhaut, die zum Zweck der Sehpurpurextraktion wie gewöhnlich im Dunkeln unter schwachem Rotlicht behandelt wird. Wie kann man dabei versichern, ob eine abpräparierte Netzhaut pigmentfrei ist oder nicht? Bei gewöhnlicher Sehpurpurextraktion durch mehrstündiges Einlegen der Netzhäute in eine Lösung von Extraktionsmitteln, die eigentlich an allen Netzhautgeweben zytolytisch wirken, müssen auch grewisse Substanzen aus den zertrümmerten Pigmentzellen (resp. Pigmentkörnern) in den Extrakt übergehen. Der Gedanke wäre deshalb sehr natürlich, dass die Sehpurpurregeneration durch den Gehalt des Extraktes an solchen „Pigmentepithelstoffen“ mehr oder minder beeinflusst wird. Der Pigmentgehalt der abpräparjerten Netzhaut, und folglich der Gehalt des Sehpurpurextraktes an Pigmentepithelstoffen, ist nicht nur nach der Tierart und der Jahreszeit, sondern besonders nach der Behandlungsweise sehr verschieden. Und das Versuchsresultat bezüglich der Sehpurpurregeneration muss je nach dem Gehalt solcher Substanzen sehr verschieden ausfallen.

Wir konnten in den folgenden Versuchen, die von Januar bis Dezember 1935, also alle Jahreszeiten hindurch, ausgeführt wurden, einmal nach der gewöhnlichen und nach der Saitoschen Methoder) praktisch von Pigmentepithelstoffen freie, ein andermal aber nach eigener Methode an solchen Stoffen reiche Sehpurpurextrakte herstellen und damit die Regeneration des Sehpurpurs vergleichend untersuchen. Wir sind dabei zu interessanten Resultaten gekommen, die zur Lösung der oben angeführten Frage beitragen können, worüber wir hier mitteilen wollen.

I. Versuche mit dem Sehpurpurextrakt, der keine oder sehrgeringe Pigmentepithelstoffe enthält.

\section{Versuchsmethode.}

1. Herstellung des Sehpurpurextraktes.

a) Gewölnliche Methode (Vgl. Ho soya und Sa ito $0^{8}$ ). In Dunkelkammer 
wurden unter rotem Licht (über $650 \mathrm{~m} \mu$ ) die Netzhäute von Fröschen (Rana nigromaeulata) abpräpariert und davon zwanzig ausgewählt, die unter dem Rotlicht keine sichtbare Pigmentanhaftung zeigten, in $1 \mathrm{ccm} 2 \%$ iger Lösung von Na-glykocholat gebracht, 5-6 Stunden bei $18-20^{\circ} \mathrm{C}$ stehen gelassen, dann mit 4800 Touren pro Min. 30 Minuten lang zentrifugiert. Auf diese Weise wird ein sehr klarer Extrakt gewonnen. Im abzentrifugierten Bodensatz finden sich mikroskopisch stets Fuszinkörner, wenn auch in ganz geringer Menge.

b) Die Methode von Z.Saito ${ }^{7}$. Aus 20 Dunkelnetzhäuten von Fröschen wird durch $1 \mathrm{ccm}$ von $45 \%$ Rohrzuckerlösung das ,Stäbchenaussengliederpräparat" isoliert. Aus diesem wird durch den Zusatz von 0,5 ccm $2 \%$ iger Na-glykocholatlösung innerhalb einiger Minuten ein sehr klarer Sehpurpurextrakt gewonnen. Von diesem lässt sich durch Zentrifugieren kein Bodensatz mehr abscheiden. Zu einmaliger Untersuchung wurden zwei Portionen solcher Extrakte, also $1 \mathrm{ccm}$, gebraucht.

\section{Untersuchung der Regenerationserscheinung.}

Jeder Sehpurpurextrakt in einer Menge von $1 \mathrm{ccm}$ wird unter rotem Licht in drei Teile geteilt und jeder einzeln in einen Absortionstrog* von Leybold gebracht. Ein Teil davon wird als Kontrolle aufbewahrt, die zwei anderen ( $I$ u. II) wie unten untersucht:

a) Zuerst wird mit dem Teil I die spektrale Absorption gemessen (Hosoya u. Z. Saito). Er wird nun mit einer $100 \mathrm{~V}$ u. $100 \mathrm{~W}$ Mazdalampe aus $1 \mathrm{~m}$ Abstand 30 Sekunden lang belichtet, wodurch seine Bleichung, wie dabei festgestellt wird, so weit fortschreitet, dass sein Farbenton dem zwischen $\mathrm{B}_{2}$ und $\mathrm{B}_{4}$ der $\mathrm{G}$ arten schen Tafel entspricht, d. h. nur soweit, dass es noch nicht das Sehgelb erreicht, und dann, nachdem die zweite Absorptionsmessung gemacht ist, wird der Extrakt lichtdicht bei Zimmertemperatur aufbewahrt, und nach 5 Stunden zum dritten Male die Absorptionsmessung ausgeführt, um auf seine Regeneration zu prüfen.

b) Der Teil II wird nach der ersten Absorptionsmessung in gleicher Weise wie Teil I, aber 5 Minuten lang, belichtet, wodurch er, wie nachweislich zu Sehgelb, d. h. zu $F_{1}$ der Garte nschen Tafel, gebleicht wird. Darauf wird die zweite Absorptionsmessung gemacht. Die Aufbewahrung geschieht nun wie von Teil I im Dunkeln, und nach 2 Stunden wird die dritte und nach weiteren 3 Stunden die vierte Absorptionsmessung: ausgeführt.

Die Absorptionsmessungen haben wir in den meisten Fällen nur in drei Wellenlängen, nämlich 640,500 und $430 \mathrm{~m} \mu$, ausgeführt, und zwar (1) aus dem Grunde, dass die Absorptionsänderung bei der Bleichung gewöhnlich bei 500 einerseits und 640 und $430 \mathrm{~m} \mu$ andererseits in entgegengesetzter Richtung statt- 
findet und deshalb für die Erkennung der Regeneration die Messungen an diesen drei Stellen genügen können, und (2) dass wir etwaige Bleichwirkungen der sehr kurzzeitigen Belichtung für die spektrale Messung möglichst vermeiden wollten, wenn auch eine einmalige Messung wegen des kurzperiodischen CambridgeSaitengalvanometers sehr schnell innerhalb einiger Sekunden geschehen konnte. Aber die Messungen am Ende des Versuches nach 5 stündiger Dunkelaufbewahrung sowie die Kontrollmessungen haben wir stets in 8 Wellenlängen in der Reihenfolge : 500-480-440-420-520-550-600-640 mp gemacht.

\section{Versuchsresultate.}

Die in Fig. 1 und 2 angegebenen Resultate entsprechen der Extraktionsmethode (a), und die in Fig. 3 und 4 der Methode (b). Aus den Fig. 1 u. 3 ersieht sich, dass die Absorptionsgrösse $\mathrm{E}$ in $500 \mathrm{~m} \mu$ nach der Belichtung von 30 Sekunden eine Abnahme von $12 \%(0,550-0,483=$ $0,067)$ resp. 13\% $(0,482-0,418=0,064)$, und nach 5 Stunden Dunkel-

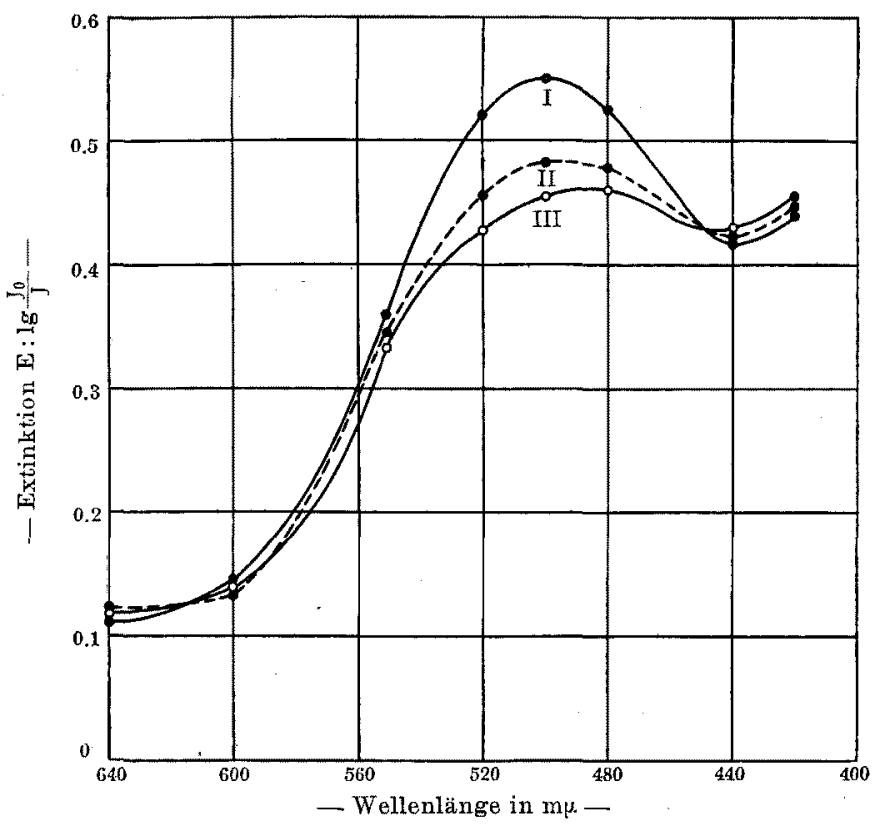

Fig. 1. Sehpurpurextrakt hergestellt nach der gewöhnlichen Methode (nur geringe Pigmentepithelstoffe enthalten). I: Anfangsabsorption, II : Absorption nach 30 sekundiger Belichtung, IIT : Die nach 5stündiger Dunkelaufbewahrung. 
aufbewahrung eine weitere Abnahme (bis 0,455 resp. 0,387), die Nachbleichung, zeigt. Nach den Fig. 2 und 4 fand nach 5 Min. langer Belichtung eine sehr starke Absorptionsabnahme von $44 \%(0,550-0,308$ $=0,242)$ resp. $55 \%,(0,486-0,220=0,260)$ und nach der Dunkelaufbewahrung eine weitere Abnahme statt. Auch im Violettgebiet ist die Absorptionsveränderung erkennbar, die für die fortschreitende Bleichung charakteristisch ist. Also ist in diesen beiden Arten von Extrakten während der Dunkelaufbewahrung keine Andeutung für die Regeneration vorhanden, sondern nur eine Nachbleichung.

Nebenbei sei bemerkt, dass sich der Charakter der Absorptionskurve und ihrer Veränderung bei der Bleichung in den Fig. 1 und 2 einerseits von dem in 3 und 4 andererseits deutlich unterscheidet. Dies beruht, wie von Z. Saito darauf hingewiesen ist, auf der Versehiedenheit der Reinheit der Extralte, die bei der Sa ito schen Methode viel vollkommener ist als bei der gewöhnlichen Methode.

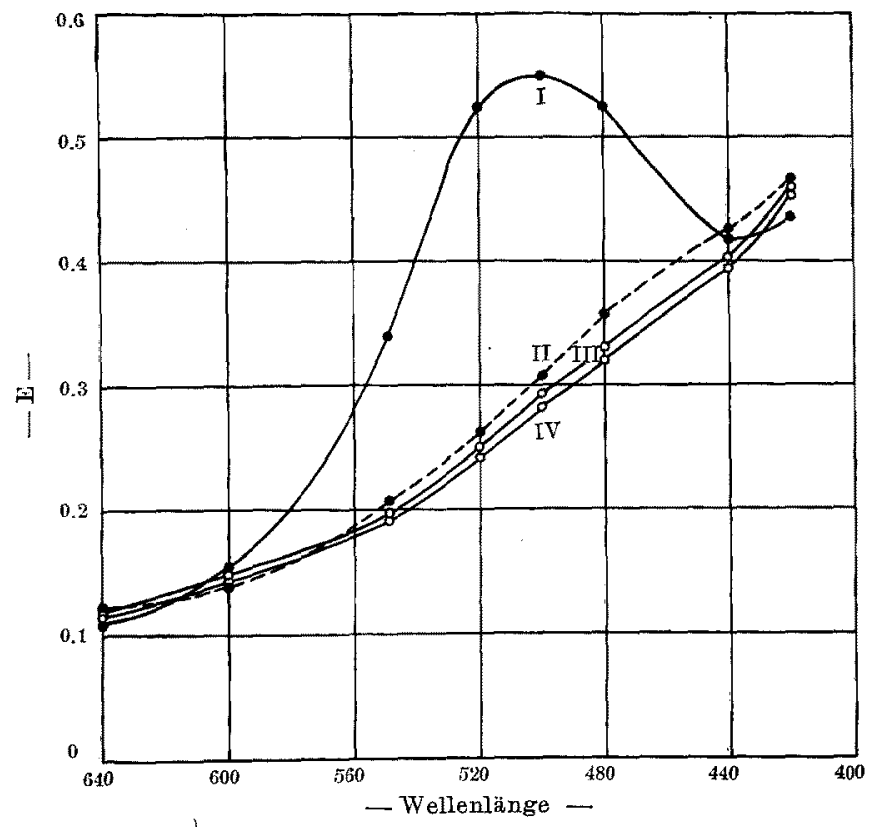

Fig. 2. Sehpurpurextrakt hergestellt nach der gewöhnlichen Methode (nur geringe Pigmentepithelstoffe enthalten). I: Anfangsabsorption, II : Absorption nach 5minutiger Belichtung, III : Die nach 2-, und IV: nach weiterer 3stündiger Dunkelaufbewahrung. 


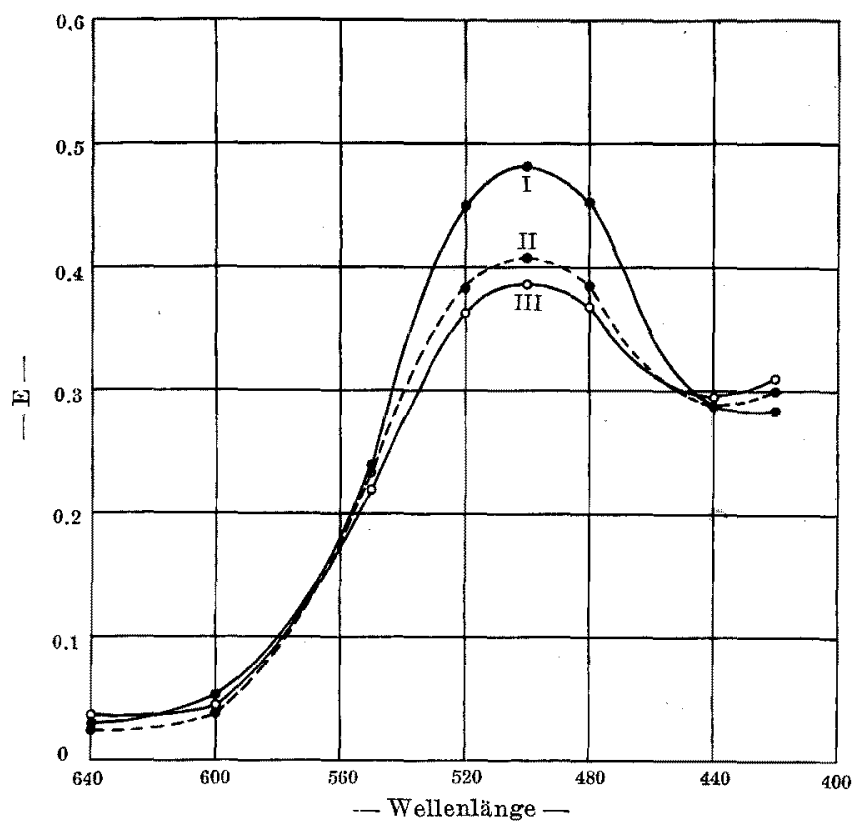

Fig. 3. Sehpurpurextrakt hergestellt nach der Methode ron Z. Saito (keine Pigmentepithelstoffe enthalten). Wie bei Fig. 1.

II. Versuche mit dem Extrakt, der ans Pigmentepithel und Stäbchenaussengliedern hergestellt wird.

\section{Methode.}

Wenn man eine abpräparierte Froschnetzhaut mit nach oben gekehrter Sehepithelschichtseite auf ein Objektglas auflegt und auf weissem Papier bei schwachem Tageslicht beobachtet, so findet man, dass die Netzhaut nicht in ihrer ganzen Ausdehnung gleichmässig purpurn aussieht, sondern mit purpurfreien Stellen fleckenweise bestreut erscheint. Wenn man nun dieses Präparat bei schwachem Licht und minimaler Irisweite in ca. 50 facher Vergrösserung mikroskopiert, so erkennt man, dass entsprechend den purpurfreien Stellen die Stäbchenaussenglieder von Innengliedern ausgerissen sind. Sie müssen mit dem Pigmentepithel zusammen an der Chorioidea anhaften geblieben sein. Nun haben wir aus 40 Exemplaren von den hinteren Bulbushälften, von denen die Netzhäute schon abgetragen waren, das Pigmentepithel (zu- 


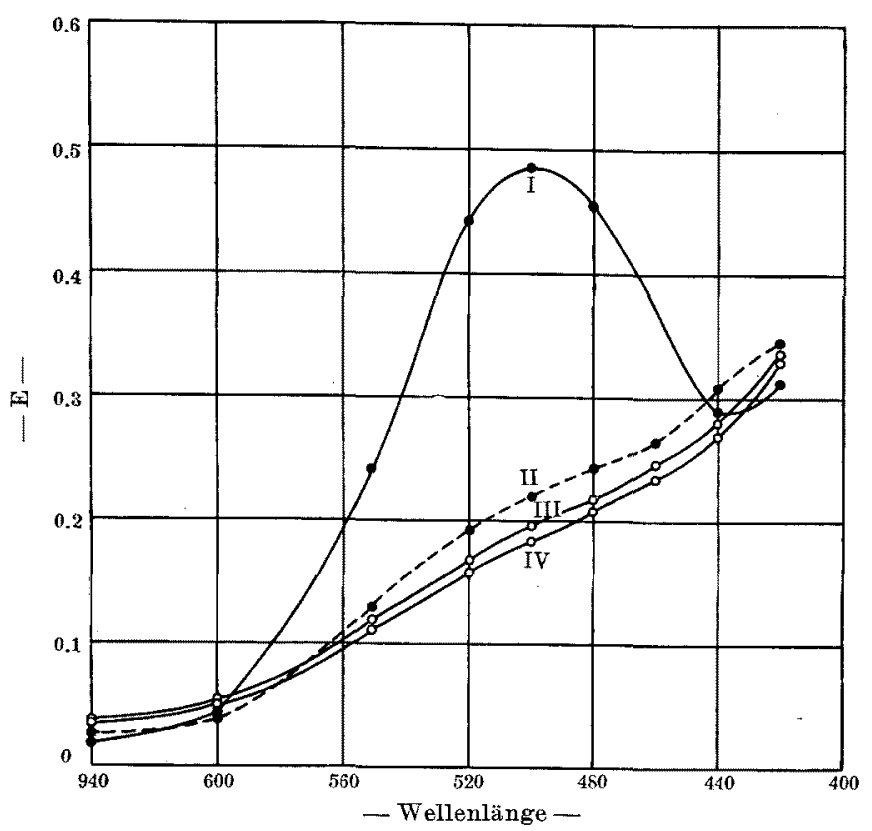

Fig. 4. Sehpurpurextrakt hergestellt nach der Mothode von $Z$. Sa ito (keine Pigmentepithelstoffe enthalten). Wio bei Fig. 2.

sammen mit den daran anhaftenden Aussengljedern) mit einem kleinen gekrümmten Glasstab herausgekratzt, ohne indessen sonstige Gewebe zu zerreissen, und in die physiologische Kochsalzlösung hineingebracht, dann mit 4800 Touren pro Min. 30 Minuten lang zentrifugiert und den abgeschiedenen Bodensatz mehrere Stunden in $1 \mathrm{ccm}$ $2 \%$ iger Na-glykocholatlösung gelegt und mit 6000 Touren pro Min. 30 Minuten lang zentrifugiert. Durch diese Prozedur konnten wir einen Sehpurpurextrakt gewinnen, der reich an den Stoffen ist, die aus dem Pigmentepithel stammen. Fuszinkörner sind aber in 2\%iger Glykocholatlösung unlöslich, deshalb durch das Zentrifugieren entfernt und im Extrakt nicht enthalten. Der so gewonnene Extrakt erscheint stärker getrübt als der durch gewöhnliche Methode oder nach Saito hergestellte.

An diesem an Pigmentepithelstoffen reichen Sehpurpurextrakt wurden spektrale Messungen wie bei der I. Versuchsreihe ausgeführt. 


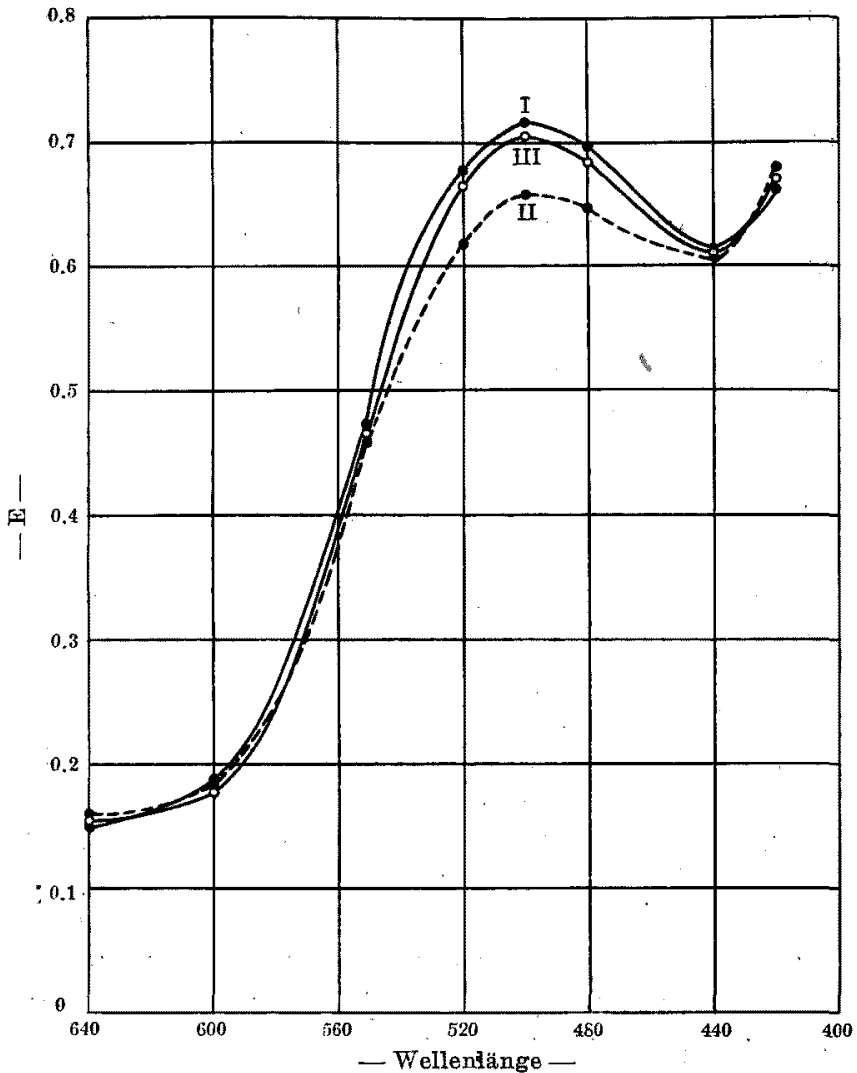

Fig. 5. Sehpurpurextrakt hergestellt nach eigener Methode (grosse Menge von, Pigmentepithelstoffen enthalten). Wie bei Fig. 1.

\section{Resultate.}

Ans Fig. 5 und Tabelle a ist ersichtlich, dass die spektrale Absorption nach 30 Sek. Belichtung, also bei der Bleichung zu $\mathrm{B}_{1}-\mathrm{B}_{3}$ der Gartenschen Tafel, eine dieser Bleichung entsprechende Veränderung erfahren hat, sie aber nach 5stündiger Dunkelaufbewahrung fast zum Anfangswert zurückgekehrt ist, dass also die Regeneration deutlich stattgefunden hat. 'Die Absorption in $500 \mathrm{~m} \mu$ nahm nach der Belichtung um $8 \%(0,719-0,661=0,058)$ ab und ging nach 'der Dunkelaufbewahrung um $6 \%(0,661-0,703=-0,042)$ zurück. In einem anderen Versuche (Tabelle b), wobei die Belichtung I Minute gedauert 


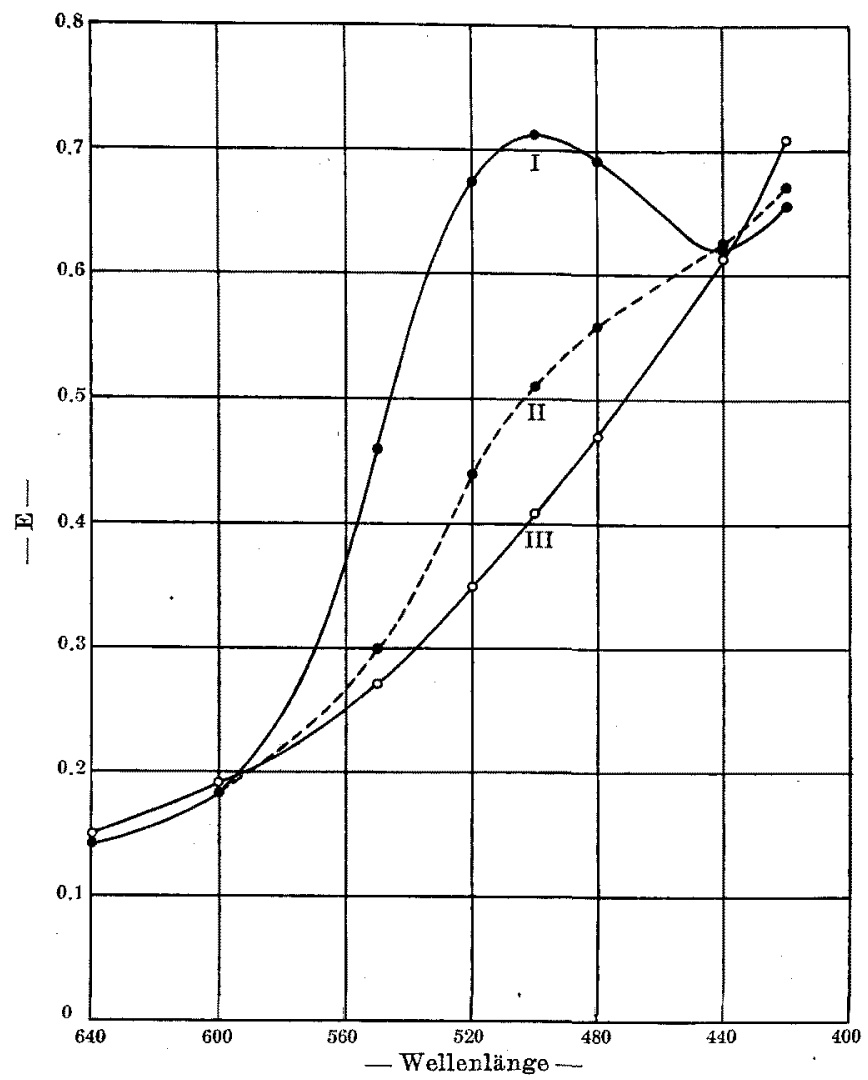

Fig. 6. Sehpurpurextrakt hergestellt nach eigener Methode (grosse Menge von Pigmentepithelstoffen enthalten). Wie bei Fig. 2, aber Kurve III: Absorption nach 5stündiger Dunkelanfbewahrung.

hat, war die Absorptionsabnahme nach der Belichtung 12\%, $(0,721-$ $0,637=0,084)$ und die Wiederzunahme $7 \%(0,637-0,685=-0,048)$. Hierbei ist also eine relativ geringere Regeneration nachweisbar.

Da in diesen Extrakten grössere Mengen von Fremdstoffen enthalten sind, wodurch die charakteristische Absorptionskurve bedingt wird, und auf diese Stoffe ein grosser Anteil der starken Absorption dieser Extrakte zurückzuführen ist, so kann der dem Sehpurpur angehörige Anteil der Ab- und Zunahme der Absorption bei der Belichtung und Regeneration relativ grösser sein.* Jedoch darf man nicht übersehen,

* Man beachte, dass die absolute Absorptionsabnahme in Fig. 1 und 30,067 resp. 0,064 und in Fig. 5 0,058 beträgt, also zwisehen beiden keine grosse Differenz existiert. 


\section{Tabelle.}

Spektrale Absorption des Sehpurpurextraktes vor und nach 30sekundiger (a) resp. 1minutiger (b) Belichtung und nach dara uffolgender 5stündiger Dunkelaufbewahrung.

\begin{tabular}{|c|c|c|c|c|c|c|c|c|c|}
\hline \multirow[b]{3}{*}{ a. } & \multirow[b]{2}{*}{$\begin{array}{l}\text { Wellen- } \\
\text { Iänge }\end{array}$} & \multicolumn{3}{|c|}{ Spektrale Absorption (E) } & & \multirow[b]{2}{*}{$\begin{array}{l}\text { Wellen- } \\
\text { länge }\end{array}$} & \multicolumn{3}{|c|}{ Spektrale Absorption (D) } \\
\hline & & $\begin{array}{l}\text { vor } \\
\text { Beli }\end{array}$ & $\begin{array}{l}\text { nach } \\
\text { ung }\end{array}$ & $\begin{array}{c}\text { nach } \\
\text { Dunkel- } \\
\text { anfbe- } \\
\text { wahrung }\end{array}$ & & & $\begin{array}{l}\text { Por } \\
\text { Beli }\end{array}$ & $\begin{array}{l}\text { nach } \\
\text { ung }\end{array}$ & $\begin{array}{c}\text { nach } \\
\text { Dunkel- } \\
\text { aufbe- } \\
\text { wahrung }\end{array}$ \\
\hline & $\begin{array}{l}640 \\
600 \\
550 \\
520 \\
500 \\
480 \\
440 \\
420\end{array}$ & $\begin{array}{l}0,154 \\
0,185 \\
0,471 \\
0,680 \\
0,719 \\
0,698 \\
0,613 \\
0,661\end{array}$ & $\begin{array}{l}0,159 \\
0,187 \\
0,462 \\
0,628 \\
0,661 \\
0,647 \\
0,608 \\
0,677\end{array}$ & $\begin{array}{l}0,158 \\
0,185 \\
0,470 \\
0,665 \\
0,703 \\
0,683 \\
0,609 \\
0,670\end{array}$ & $\mathrm{~b}$ & $\begin{array}{l}640 \\
600 \\
550 \\
520 \\
500 \\
480 \\
440 \\
420\end{array}$ & $\begin{array}{l}0,15 \tilde{0} \\
0,186 \\
0,470 \\
0,682 \\
0,721 \\
0,701 \\
0,614 \\
0,668\end{array}$ & $\begin{array}{l}0,159 \\
0,187 \\
0,460 \\
0,605 \\
0,637 \\
0,624 \\
0,609 \\
0,678\end{array}$ & $\begin{array}{l}0,156 \\
0,187 \\
0,465 \\
0,649 \\
0,685 \\
0,671 \\
0,609 \\
0,665\end{array}$ \\
\hline
\end{tabular}

dass hier die Farbe des Sehpurpurs sich durch 30sekundige Belichtung noch nicht bis $\mathrm{B}_{2}-\mathrm{B}_{4}$ (Vgl.S. 449 ) wie bei der $\mathrm{I}$. Versuchsreihe, sondern nur bis $\mathrm{B}_{1}-\mathrm{B}_{3}$ bleichte, und dieser geringeren Bleichung entsprechend auch der Betrag der Absorptionsabnahme geringer war. Diese Verzögerung der Sehpurpurbleichung beruht wahrscheinlich darauf, dass die im Extrakt reichlich beigemischten Pigmentepithelstoffe eine Neigung haben, sich an einen Teil des ausgebleichten Sehpurpurs zu binden, um wieder Sehpurpur zu bilden.

Die Absorption verändert sich im kurzwelligen Gebiete natiurlich in der umgekehrten Richtung, indem sie nach der Belichtung zu-, und nach der Dunkelaufbewahrung wieder abnimmt und sich dem Anfangswert nähert.

Wenn aber die Belichtung 5 Minuten danert und die Ausbleichung: (28\% Absorptionsabnahme bei $500 \mathrm{~m} \mu$ ) $\mathrm{E}_{3}$ der Gartenschen Tafel exreicht, was ungefähr dem Sehgelb entspricht, so ist danach keine Regeneration mehx, sondern nur noch Nachbleichung nachweisbar (Fig. 6).

Aus allem geht hervor, dass der Sehpurpur, wenn in seinem Extrakt eine grössere Menge von Pigmentepithelstoffen enthalten ist, aus einer noch nicht bis zum Sehgelb vorgeschrittenen Ausbleichung während der Dunkelaufbewahrung regenerieren kann, sonst aber sowohl bei sehr geringem Gehalt an Pigmentepithelstoffen als auch nach stä2*kerer Bleichung bis zum Sehgelb keine Regeneration mehr, sondern nur eine Nachbleichung stattfindet. 
Wir können den neutralen Punkt dex Bleichung oder die Bleichungsgrenze, von wo aus weder Regeneration noch Nachbleichung vor sich geht, noch nicht bestimmen. Aber es lasst sich ans unseren Resultaten vermuten, dass er bei etwas mehr als $20 \%$ Absorptionsabnahme bei $500 \mathrm{~m} / \mathrm{l}$ liegt.

\section{Eröxterung der Resultate.}

Nach dem Abschluss unserer Versuche ist die Mitteilung von Hecht, Chase, Shlaer und Haig ${ }^{5 / 5}$ über die gelungene Sehpurpurregeneration in der Lösung erschienen. Nach ihnen wird die Regeneration einerseits durch die Stärke der Belichtung resp. den Grad der Bleichung bedingt und andererseits durch $p \mathrm{H}$ des Extraktes. Die Regeneration findet nämlich nur bei einer geringeren Bleichung statt, und zwar wenn die Absorptionsabnahme in 500 m/ geringer ist als 15 $\%$. Bei einer stärkeren Absorptionsabnahme als $20 \%$ ist keine Regeneration mehr, sondern nur eine Nachbleichung zu konstatieren. Sie fanden diese Regeneration nur bei $p H$ von 8-6,6. In neuester Zeit hat Sigesad a) den Versuch von Hecht $u$. a. nachgeprüft und will die Regeneration sogar auch nach der Bleichung zum Sehgelb gefunden haben. Bezüglich des Einflusses von $p \mathrm{H}$ ist er zu demselben Resultat wie $\mathrm{H}$ ech t u. a. gekommen.

Der Betrag der Ausbleichung bei Hech t u. a., von der aus die Regeneration noch stattfinden kann, stimmt mit unserem Resultat sehr gut tiberein. Bezüglich der $p \mathrm{H}$ haben wir keine besondere Untersuchung gemacht; wir arbeiteten nur unter der neutralen Reaktion ( $p H$ von 7,1 bei $16^{\circ} \mathrm{C}$ ). Uns exscheint aber die von $\mathrm{Hecht} u$. a. angegrebene $p \mathrm{H}$-Grenze für die Sehpurpurregeneration von Haus aus als ein natiurliches Resultat. Denn es ist von Minoshima und Nozaki ${ }^{10)}$ schon nachgewiesen und auch unserer Erfahrung nach sicher, dass der Sehpurpur ausserhalb der $p H$-Grenze von 8-5 auch ohne Lichtwirkung, d.h. im Dunkeln selbst, verbleicht. Es ist also selbstverständlich, dass dabei keine Regeneration stattfinden kann.

Es ist zu bedauern, dass Hecht und seine Mitarbeiter sowie Sigesada in ihren Untersuchungen nicht berücksichtigt haben, ob vielleicht in den von ihnen hergestellten Sehpurpurextrakten, wenn auch Pigmentkömer sorgfältig ausgeschaltet wurden, nicht noch gewisse von Pigmentepithel herstammende Substanzen enthalten waren, deren grosse Bedeutung für die Sehpurpuregeneration, glauben wir, von uns zum ersten Male versichert werden konnte.

Zuletzt noch ein Wort über die Nachbleichung! Hecht und seine Mitar- 
beiter sowie besonders W ald behaupten, dass die Nachbleichung nur bei frischem Sehpurpurextrakt, aber nicht mehr nach einer Dunkel- und Kaltaufbewahrung von einer Woche Dauer zu beobachten ist. Doch ist in der W ald schen Kurve nach einwöchiger Aufbewahrung noch eine, wenn auch geringe Nachbleichung erkennbar. Auch nach unserer Erfahrung vermindert sich die Nachbleichung mit dem Veralten des Extraktes, wie sie z. B. sehon 24 Stunden nach der Extraktion nicht mehr so deutlich ist wie beim frischen Extrakt.

\section{Zusammenfassung.}

Die Regeneration des von der Netzhaut extrahierten Sehpurpurs wurde mit besonderer Rücksicht auf den Gehalt des Extraktes an Stoffen, die aus dem Pigmentepithel stammen, untersucht, was bis jetzt völlig vernachlässigt geblieben ist. Es ergab sich folgendes :

1. Der Sehpurpur im Extrakt mit $p H$ von 7,1 , der nach Z. Saito aus den isolierten Stäbchenaussengliedern hergestellt wird, also keine Pigmentepithelstoffe enthält, oder in gewöhnlicher Weise direkt aus der Netzhaut gewonnen, aber mit besonderer Sorgfalt von sichtbaren Pigmentepithelteilen befreit wirde, regeneriert während der Dunkelaufbewahrung nicht, selbst wenn die Bleichung noch nicht das Sehgelb erreicht, sondern zeigt nur eine weitere Bleichung (Nachbleichung).

2. In einem Extrakt, der in besonderer Weise aus den Stäbchenaussengliedern samt dem Pigmentepithel hergestellt wird, der also gewisse aus dem Pigmentepithel herkommende Stoffe (ausgenommen Fuszinkörner) reichlich enthalten muss, regeneriert der Sehpurpur aus einem gebleichten Zustand, der noch nicht zum Sehgelb geführt ist, und zwar um so vollständiger, je weniger die Bleichung vorgeschritten ist. Nach einer stärkeren Bleichung findet aber auch bei diesem Extrakt keine Regeneration mehr statt, sondern nur eine Nachbleichung.

Aus diesen Ergebnissen kann geschlossen werden, dass der Sehpurpur nicht nur in der Retina, sondern auch im Extrakt unter bestimmten Bedingungen aus einem Bleichungszustand regenerieren kann, und dass für diese Regeneration sowohl in der Netzhaut als auch im Extrakt das Vorhandensein gewisser Stoffe aus dem Pigmentepithel, nicht aber das von Pigmentkörnern selber, eine notwendige Bedingung darstellt.

\section{Literatur.}

(1) A. F wa ld u. W. Küh ne, Unters. a. d. Physiol. Inst. Heidelberg, 1878, 1, 248. 
(2) S. Garten, v. Graefes Arch. f. Opthalm., 1906, 63, 112.

(3) N. Andogsky, r. Graefes Areh. f. Opthalm., 1897, 44, 404.

(4) Y. Hos oy a, Pflügers Arch. f. Physiol., 1933, 233, 57.

(5) S. Hecht, A. M. Chase, S. Shl aer \& C. Haig, Science, 1936, 84, 331.

(6) G. Wa ld, Nature, 1937, 139,587.

(7) Z. Saito, Tohokn J. Exp. Med. 1938, 32, 432.

(8) Y. Hos o y a u. Z. Saito, Tohoku J. Exp. Med., 1935, 27, 172.

(9) K. Sigesada, Nihon Seiri. Z., 1937, 2, 114.

(10) T. Minoshima u. S. Nozaki, Proc. Jap. Physiol. Soe. 1935 (Jap. J. of Med. Sei, III, Biophsics, 4) 79* 